\title{
EL TOPICO DE LA DEBILIDAD ASOCIATIVA ANDALUZA DESDE LA ANTROPOLOGIA SOCIAL: EL CASO DEL ALJARAFE
}

\author{
Javier ESCALERA REYES*
}

Un rasgo con el que se ha caracterizado de modo genérico a la sociedad andaluza ha sido la debilidad, al menos aparente, que tradicionalmente habrían tenido en ella las tendencias asociativas en comparación con otras sociedades, incluso dentro del mismo estado español. Situación que, siendo cierta en parte, en absoluto alcanza las proporciones que se le han llegado a atribuir, presentando además componentes y aspectos explicados fundamentalmente en conexión con los condicionamientos socioeconómicos básicos y con algunos de los marcadores profundos que definen y configuran lo andaluz, la etnicidad andaluza. En relación con ello pretendemos hacer notar la importancia de las funciones socio-políticas de unas formas asociativas como los casinos, peñas y sociedades recreativas, que, como en el caso de las hermandades y cofradías, han sido menospreciadas, cuando no absolutamente olvidadas, en determinados análisis sobre el asociacionismo andaluz, debido principalmente a la aplicación de modelos extraños a nuestra realidad sociocultural.

\section{EL ASOCIACIONISMO VOLUNTARIO: \\ ESTADO DE LA CUESTION E HIPOTESIS INTERPRETATIVAS}

El ser humano es esencialmente un animal sociable, es decir que para el desarrollo de sus potencialidades y capacidades como miembro de su especie precisa de la interrelación con otros individuos con los que formar una comunidad, compartiendo y comunicándose sus vivencias, experiencias, sentimientos e inquietudes, lo que se conoce bajo el término sociabilidad ${ }^{1}$. Esta se ex-

* Profesor Titular de Antropología Social.

1. Sobre el concepto de sociabilidad ver: BOZON, M. «La fréquentation des cafés dans une petite ville ouvrière». Ethnologie Française. XII, n. ${ }^{\circ} 2$, 1982, pp. 137-142; y AGULHON, M. Etude d'une mutation de sociabilité. Le Cercle dans la France Burgeoise (1810-1848). París, 1977. 
presa y desarrolla en ámbitos diferentes según el nivel de complejidad y desarrollo sociocultural de cada sociedad. En las sociedades preindustriales el marco para ello es ofrecido generalmente por instancias no específicamente destinadas a este fin, como puedan ser los grupos familiares y de parentesco, los grupos corporativos, los gremios, etc. Pero en las sociedades urbano-industriales dichas instancias se verán profundamente erosionadas merced a la actuación de diversos factores: ruptura de los vínculos de parentesco, complejización de los sistemas de relaciones sociales, de las pautas de comportmaiento y la tecnología; el desarraigo de amplios sectores de la población debido a la concentración en grandes núcleos urbanos de individuos procedentes de las zonas rurales y campesinas, entre otros muchos. Frente a la disolución de los marcos tradicionales para la expresión de la sociabilidad surgen nuevas formas, constituidas principalmente por las correspondientes a lo que se ha dado en denominar asociacionismo voluntario ${ }^{2}$, las cuales proporcionarán los ámbitos en los que los individuos podrán encontrar satisfacción a la necesidad humana de comunicación e interrelación, de expresión de la afectividad, de la búsqueda de apoyo y seguridad en otros individuos para objetivos y finalidades diversos: cooperación, prestigio, poder.

Sobre la base de la división en clases sociales, siempre presente y determinante en las sociedades urbano-industriales y sus entornos rurales y campesinos, y sobrepasando los límites de las debilitadas relaciones familiares y/o de parentesco, existe toda una variedad de formas de asociación a través de las cuáles, más allá e incluso antes de sus propias finalidades explícitas concretas, se expresa y desarrolla la sociabilidad generalizada y todo un rico entramado de relaciones interpersonales e intercambios de apoyo mutuo y alianzas entre sus miembros. Se trata pues de asociaciones y grupos de carácter voluntario, al menos en teoría, en los que se combinan y entrecruzan gran número de elementos y aspectos que configuran una determinada unidad social y que se ponen de manifiesto en su funcionamiento cotidiano: la identidad o el antagonismo de clase, las redes sociales, las relaciones de poder, los sistemas ideológicos, las actividades económicas, así como otros aspectos más generales, como el espíritu gregario, la tendencia lúdica, la necesidad de expresión de la afectividad, el «instinto» de búsqueda de protección en el grupo, etc., todo lo cuál las convierte en importantísimos vehículos de canalización de la actividad y la participación social de los individuos y los grupos.

2. Para una visión general sobre los estudios socio-antropológicos que han centrado su interés sobre el asociacionismo voluntario, ver: BANTON, M. «Asociaciones voluntarias I: Aspectos Antropológicos». Enciclopedia Internacional de las Ciencias Sociales. Madrid, 1974. Vol. 16; SILLS, D.L. «Asociaciones voluntarias II: Aspectos sociológicos». Ibidem; y sobre todo SMITH, C. y FREEDMAN, A. Voluntary Associations. Perspectives on the Literature. Cambridge, 1972 . 
La voluntariedad de estas formas asociativas debe ser considerada siempre de manera relativa, sobre todo en comunidades de pequeño tamaño, en las que la teórica libertad de elección y afiliación por parte de los individuos en las mismas, se ve frecuentemente condicionada o limitada de hecho por factores como los vínculos y relaciones familiares, las interrelaciones personales, los lazos de dependencia y patronazgo, etc., todo lo cuál se ve potenciado por el estrecho control social que impera en este tipo de comunidades, en las que las relaciones personales directas y continuadas entre sus miembros actúan como factor determinante, dado que los individuos se encuentran en contacto permanente, aún desempeñando una gran variedad de roles; es decir que, dada la reducida dimensión de la sociedad local, los individuos se ven obligados a relacionarse continua, sucesiva y a veces simultáneamente con los mismos individuos en el desempeño de sus diferentes roles sociales, con lo que resulta prácticamente imposible establecer una separación entre las situaciones y comportamientos que teóricamente corresponderían a cada relación de rol, quedando unas y otras teñidas inevitablemente por las demás. Las relaciones de rol instrumentales quedan de esta forma personalizadas y cargadas de componentes afectivos.

El campo del asociacionismo voluntario presenta una gran amplitud y ambigüedad en su delimitación, incluyéndose dentro del mismo, según la tradición antropológica, todo tipo de agrupación o asociación no basado en el parentes$c o$. Indefinición ante la cuál se hace preciso a nivel operativo, sobre todo cuando se plantea su estudio en el seno de sociedades complejas, restringir el campo del mismo a aquellas formas de asociación no ligadas directa o indirectamente a los sistemas de organización y a las instituciones que integran las estructuras del Estado (iglesias, partidos, sindicatos, etc.) y que no aparecen relacionadas en primera instancia con los sistemas de producción y las estructuras económicas de dichas sociedades, como plantea D.L. Sills ${ }^{3}$.

En cuanto a las funciones del asociacionismo voluntario también existe una gran diversidad de interpretaciones, desde las que lo consideran esencialmente como un mecanismo de adaptación frente al cambio sociocultural, hasta las que le adjudican un carácter eminentemente instrumental. Nosotros pensamos con M.T. Walker, J. Hanson, D.L. Sills y otros ${ }^{4}$ - y este es uno de los presupuestos centrales de nuestro análisis- que en las sociedades complejas «desa-

3. BANTON, M. Op. cit. pp. 611-612; SILLS, D.L. Op. cit. pp. 615-616; SMITH, C. y FREEDMAN, A. Op. cit. pp. V-VIII y 1-32.

4. ROSE, A.M. "Voluntary Associations under conditions of competition and conflict». Social Forces. Vol. 34, 1955; SILLS, D.L. «Voluntary Associations: Instruments and objets of change». Human Organization. Vol. 18 , n. ${ }^{0} 1$, 1959, pp. 17-21, y Op. cit. 1974; WALKER, M.T. y HANSON, J. «Voluntary Associations of Villalta: Faliure with a purpose». Human Organization. Vol. 37, n. $^{\circ} 1$, 1978, pp. 64-68. 
rrolladas» este asociacionismo presenta, en primer lugar, unas características y una diversidad de formas que lo hacen diferenciarse claramente del que se puede dar en situaciones de cambio sociocultural profundo, donde si parece fundamental su función adaptativa; y en segundo lugar, que aunque en las citadas sociedades pueda seguir ofreciendo un mecanismo de adaptación frente al cambio de las estructuras «tradicionales», esta no es su función principal, sino que por el contrario las asociaciones voluntarias constituyen elementos muy importantes dentro de los sistemas de relaciones sociales y de poder, así como instancias de acción para los individuos y grupos en la lucha por el control del poder, convirtiéndose incluso, en algunas ocasiones, en instrumentos o vehículos del cambio social.

Es cierto, por otra parte, que asociaciones o grupos de intereses comunes independientes de los vínculos de parentesco han existido y existen en otras sociedades con niveles de desarrollo sociocultural preindustriales ${ }^{5}$, pero según lo que conocemos de ellas carecían de muchos de los rasgos que definen de modo esencial al asociacionismo voluntario en las sociedades complejas urbano-industriales.

Como ponen de manifiesto M. Agulhon y J.Q. Wilson ${ }^{6}$, el asociacionismo voluntario propiamente dicho es un producto de la liquidación de la sociedad estamental del Antiguo Régimen y de la consolidación del modo de producción capitalista, con la burguesía como clase dominante, el mercado y el sistema democrático de representación político-institucional, que se operará en las sociedades occidentales en las que primero se produce la transición hacia formaciones económico-sociales industirales-capitalistas. Aunque con anterioridad a la Revolución burguesa se den ya algunas formas asociativas de «transición», como los clubes políticos en Gran Bretaña y los Estados Unidos de Norteamérica, o las chambrettes francesas, se trata de asociaciones relativamente poco numerosas todavía en el siglo XVIII, y que corresponderán exclusivamente a círculos muy restringidos de la burguesía en ascenso o de la aristocracia ilustrada. Será a partir de fines del siglo XVIII y principio del XIX, pero sobre todo desde mediados de este último, cuando dicho tipo de asocia-

De especial relevancia a este respecto son las recientes contribuciones al estudio del asociaciomismo voluntario desde una perspectiva más amplia que la tradicional, como por ejemplo los trabajos de antropólogos, sociólogos rurales e historiadores franceses, entre los que cabe destacar: AGULHON, M. y BODIGUEL, M. Les associations au village. Le Paradou, 1981; BO$\mathrm{ZON}, \mathrm{M}$. Vie quotidienne et rapports sociaux dans une petite ville de province. La mise en scène des différences. Lyon, 1984; GUTWIRTH, J. «Les associations de loisir d'une petite ville, Châtillon-sur-Seine». Ethnologie Française. Vol. II, n. ${ }^{0} 1-2,1972$, pp. 141-180.

5. ANDERSON, R.T. «Voluntary Associations in History». American Anthropologist. Vol. 73, n. ${ }^{\circ} 1,1979$, pp. 209-222.

6. AGULHON, M. Op. cit. 1977; WILSON, J.Q. «Clubs políticos», Enciclopedia Internacional de las Ciencias Sociales. Madrid, 1974. 
ciones empiece a generalizarse y a abrirse a, o a ser reproducidas por, otros sectores sociales, comenzando a surgir nuevas formas asociativas, como los círculos, chambres, sociedades musicales, gimnásticas, deportivas, de ayuda mutua, asociaciones de alumnos, de quintos, cooperativas, mutualidades, sindicatos agrícolas o industriales, asociaciones patronales y otras múltiples formas de asociación, correspondientes a distintos sectores sociales (asociaciones burguesas, obreras, urbanas, campesinas), formas ya plenamente características del asociacionismo voluntario «moderno», el conjunto de las cuáles va a convertirse desde entonces en el sistema de agrupamiento y organización de la sociabilidad más importante y significativo de las sociedades urbano-industriales capitalistas ${ }^{7}$.

En el caso español el retraso en la cristalización del sistema capitalista y de la división territorial del mismo operado en el seno del estado, determinará el tardío surgimiento de formas asociativas de carácter voluntario «moderno». $\mathrm{Al}$ igual que en los casos antes citados, pero de manera aún más restringida y con un carácter mucho más estrechamente ligado a la clase dominante de la sociedad estamental, con anterioridad al siglo XIX encontramos también algunas entidades como las Academias o, sobre todo, las Sociedades Económicas, que presentan algunos rasgos que las diferenciaban de las formas de agrupamiento características del Antiguo Régimen, pero cuyos integrantes solían ser miembros de la aristocracia o de la alta burguesía integrantes con frecuencia de los círculos ilustrados, pero implicados, en definitiva, en los intentos de mantenimiento de las estructuras y sistemas socioeconómicos del Estado del Antiguo Régimen, a través de su reforma y «racionalización».

Hasta 1835 no se constituye formalmente una asociación que podamos considerar ya plenamente integrada dentro del asociacionismo voluntario moderno, se trata del Casino de Bilbao. En 1836 lo hará el de Madrid, y a partir de aquí este tipo de asociaciones comenzará a extenderse por numerosos pueblos y ciudades de todo el estado, aunque a través de un proceso lento. No obstante, además, la diversidad de formas asociativas seguirá siendo muy reducida, limitándose prácticamente a las de carácter formal «recreativo-cultural», como los citados casinos y círculos.

El asociacionismo obrero sólo empezará a desarrollarse, y ello de forma clandestina durante bastante tiempo, desde bien entrada la segunda mitad del siglo XIX; el patronal no lo hará hasta principio del siglo XX. Serán las sociedades cooperativas, asistenciales y las mutualidades el tipo de entidades con mayor presencia, excepción hecha de las de carácter «recreativo-cultural» antes citadas.

7. AGULHON, M. y BODIGUEL, M. Op. cit.; SILLS, D.L. Op. cit., 1968; WILSON, J.Q. Op. cit. 
En Andalucía, como una consecuencia más del papel marginal y dependiente que le es asignado en la configuración de la división territorial del sistema capitalista español, la aparición y el desarrollo del asociacionismo voluntario moderno se producirá con mayor retraso aún que en las zonas centrales (política y económicamente) del estado.

Las primeras entidades análogas a la que surgen en dichas zonas, al menos según los datos que poseemos, serán el Casino Sevillano, creado en 1844, y el Andaluz, de 1845, extendiéndose a partir de ese momento, poco a poco, por las principales ciudades y pueblos de Andalucía ${ }^{8}$.

Además del citado retraso, el asociacionismo andaluz ha sido caracterizado por su debilidad por parte de una aproximación, ya hoy superada en parte ${ }^{9}$, que basaba su apreciación en la constatación de la reducida presencia de entidades asociativas del tipo de, y en comparación con, las existentes en otras zonas y países, fundándose para ello en criterios casi exclusivamente cuantitativos y en la aplicación en su análisis y diagnóstico del asociacionismo andaluz de modelos inadecuados a nuestra realidad.

A través de nuestra investigación hemos pretendido demostrar lo erróneo de la citada visión, centrándonos para ello en el estudio de aquellas formas asociativas que, con una dimensión, extensión e implantación menores que la hermandades y cofradías _-expresiones asociativas más representativas de Andalucía e incluso las únicas existentes en muchos lugares- ${ }^{10}$ han tenido y tienen una significación notable en la vida social de muchos pueblos y ciudades andaluces, como son las asociaciones que podrían ser clasificadas como de ocio y tiempo libre ${ }^{11}$, entidades de carácter civil, no religioso, del tipo de los casinos, círculos, peñas y sociedades recreativo-culturales.

En relación con este tipo de asociaciones partimos de la consideración de que, más allá de sus objetivos y funciones explícitos y de su carácter formal, constituyen elementos de relativa importancia en la configuración y funcionamiento de la organización social de las comunidades en las que se hallan inscritas. En primer lugar ofrecen campos o espacios sociales que posibilitan, favorecen o mantienen el establecimiento y el desarrollo de las redes de relaciones interpersonales entre los miembros de un sector más o menos amplio de la sociedad local, redes tanto de tipo vertical (relaciones de clientelismo, pa-

8. BERNAL, A.M. y LACROIX, J. «Aspects de la sociabilité andalouse. Les associations sevillanes (XIX ${ }^{c}-\mathrm{XX}^{\mathrm{c}}$ s.)». Melanges de la Casa de Velazquez, Tomo XI, Paris, 1975, pp. 435-507.

9. LINZ, J. «La realidad asociativa de los españoles». Sociología de España de los años setenta. Madrid, 1971, pp. 307-348.

10. En relación al estudio de las hermandades es obligada la referencia a los trabajos de Isidoro Moreno: MORENO NAVARRO, I. Las hermandades andaluzas, una aproximación desde la Antropologia. Sevilla, 1974; Cofradias y hermandades andaluzas: estructura, simbolismo e identidad. Sevilla, 1985.

11. DUMAZEDIER, J. y otros. Ocio y sociedad de clases. Barcelona, 1971. 
tronazgo, etc.), como horizontal (relaciones de amistad, cooperación, compañerismo, alianza, etc.). En segundo lugar, pueden ser consideradas como instrumentos, entre otros, utilizados por los grupos dominantes para ejercer, asegurar o aumentar su control sobre la comunidad, muchas veces en competencia entre individuos, familias o facciones. Así mismo, aunque con mucha menos frecuencia, dichas entidades pueden servir también como aglutinantes de los sectores desprovistos del control social y político, y pueden, en determinadas circunstancias, servir como base para oponerse al poder establecido y plantear la posibilidad de transformación de las estructuras sociales. Estructuras que, en todo caso, se ven materializadas, explicitadas, a través de dichas asociaciones, de modo mucho más claro que en el transcurso de la vida cotidiana de la comunidad, lo cuál las convierte en referencias de especial interés para el análisis de las primeras, cosa que, para nosotros, justifica por sí sola el estudio de estas formas asociativas.

Las asociaciones, como representaciones o expresiones de los sistemas de interrelación social y de los grupos existentes en una comunidad, pueden actuar también con frecuencia como elementos potenciadores y reproductores de distintos niveles de identidad: grupales, de clase, sectoriales o semicomunales, locales e incluso supracomunales, en algunos casos, actuando como elementos reafirmadores y/o recreadores de la propia identidad frente a la acción de expansión de los centros socioeconómicos, administrativos y políticos de los que dependen y ante la influencia despersonalizadora de los procesos de «modernización» que irradian desde ellos.

Como realidades sociales incardinadas en sus comunidades, las asociaciones reflejan e ilustran a la vez las transformaciones socioeconómicas y políticas experimentadas en ellas, las cuales determinan procesos de cambio, decadencia, desaparición y surgimiento de nuevas formas de asociación, según los lugares y sus características específicas, en consonancia con las nuevas condiciones de la vida social.

\section{EL ASOCIACIONISMO VOLUNTARIO EN LA SOCIOLOGIA Y LA ANTROPOLOGIA: INADECUACION DEL MODELO CLASICO AL CASO DE LAS FORMAS ASOCIATIVAS ANDALUZAS}

En la no demasiado amplia literatura antropológica sobre el asociacionismo voluntario - sobre todo en los primeros autores en abordar el estudio del mismo- suele predominar una visión de tipo funcionalista que presenta a las asociaciones esencialmente como formas de adaptación de las poblaciones «campesinas», «rurales» o de «cultura tradicional» ante el impacto desorganizador 
provocado en ellas por su progresiva integración en los procesos de extensión de la «urbanización» y la «modernización» tendentes a su asimilación y disolución por parte de las estructuras de las sociedades urbano-industriales y de los estados de los que son dependientes. Visión funcionalista, que aún hoy sigue condicionando en cierta medida el análisis del asociacionismo, procedente de su estudio en relación a las poblaciones inmigrantes engrandes centros urbanos, poblaciones integradas por individuos o grupos más o menos cohesionados, con frecuencia de origen étnico diverso y generalmente de bajo nivel de desarrollo sociocultural. Se trata la mayoría de las veces de estudios en relación a procesos de «urbanización» en países del llamado Tercer Mundo, situaciones en las que determinado tipo de asociaciones proveen de un medio de adaptación a las formas de vida urbana para estas poblaciones, una vez desarticuladas o profundamente alteradas sus formas «tradicionales» (tribales) de organización e interrelación social basadas principalmente en el parentesco ${ }^{12}$.

Según este limitado planteamiento, la complejidad consustancial a las sociedades urbano-industriales, cuyo desarrollo estuvo y está ligado a la caída de los grupos corporativos y de parentesco como formas básicas de agrupamiento y organización social, provocan en los individuos de las comunidades que experimentan procesos de transición hacia las primeras una gran confusión y sentimiento de angustia al tener que enfrentarse prácticamente en solitario a formas de vida y relaciones sociales completamente desconocidas en sus «ambientes tradicionales»; como una forma de respuesta a esa situación de indefensión e incapacidad surgirían las asociaciones, a través de las cuales se acogerían, canalizarían y controlarían las inquietudes, actividades, las luchas de dichos individuos, integrándolas en las estructuras y sistemas institucionales del estado, reflejo y cristalización de las formas de relaciones económicas, sociales y de poder características de tales sociedades. El asociacionismo se configuraría así como un plano intermedio entre los individuos y sus relaciones interperso-

12. La visión «clásica» del asociacionismo voluntario como un mecanismo esencialmente adaptativo, en situaciones de cambio sociocultural profundo, está representada principalmente por los trabajos de autores como: ANDERSON, R.T. Op. cit. 1971; «The replicate social structure». Southwestern Journal of Anthropology. Vol. 18, 1962, pp. 365-370. ANDERSON, R.T. y ANDERSON, B.G. «Voluntary Associations and Urbanization: a diachronic analysis». American Journal of Sociology. Vol. 65, 1959, pp. 265-273; «The indirect social structure of European Village Communities». American Anthropologist, n. ${ }^{\circ}$ 64, 1962, pp. 1.016-1.027; The Vanishing Village. A Danish maritime community. Seattle, 1964. HARMER, J.H. «Voluntary Associations as structures of change among the Sidamo of Southwestern Ethiopia». Anthropological Quarterly. Vol. 40, 2, Abril 1967, pp. 73-91. LITTLE, K. «The role of Voluntary Associations in Western African Urbanization». American Anthropologist. Vol. 59, 1957; West African Urbanization: A Study of Voluntary Associations in Social Change. Cambridge, 1965; «Voluntary Associations». Social Organization: Essays presented to Raymond Firth. Maurice Freeman (ed.), Chicago, 1967. 
nales y el estado y sus instituciones, desempeñando un doble papel de apoyo y protección del individuo frente a ellas, y a la vez como instrumento de control de las respuestas y comportamientos que pudieran resultar contrarios a los intereses, integridad y estabilidad del sistema, generados por los individuos abandonados en su confusión y desarraigo sociocultural.

En el medio rural que constituye el entorno sobre el que actúan y se mantienen los centros urbano-industriales, a pesar de que la desaparición de las estructuras y formas de vida tradicionales no ha sido normalmente tan brusca como en aquéllos, la influencia ejercida por los mismos sobre las comunidades campesinas ha sido fuerte y en muchos casos muy aguda, pues aunque sus miembros, dado el tamaño relativamente reducido de sus poblaciones, pueden encontrar refugio todavía en las fórmulas tradicionales de expresión de la sociabilidad, la inserción de estas comunidades en los sistemas de la economía capitalista, el mercado y la democracia política, da lugar necesariamente a un proceso de erosión de dichas formas y estructuras tradicionales. En estos casos las asociaciones desempeñan una pluralidad de funciones. Por una parte ofrecen al individuo un ámbito en el que poder identificarse, relacionarse y expresar su sociabilidad dentro de su comunidad y, lo que es muy importante, también de cara al exterior de la misma. Por otra parte estas asociaciones contribuyen a que los cambios tecnoeconómicos y socioculturales acelerados a que se ven sometidas estas comunidades se produzcan del modo menos traumático posible, actuando pues como vehículos del cambio. Pero al mismo tiempo, en buena parte de los casos, constituyen también, en cierto modo, superposiciones o reformulaciones de viejas formas de agrupamiento, corporaciones o instituciones, que mantienen, bajo formas adaptadas de las existentes en los centros urbanos, una relativa continuidad de su configuración y funcionamiento tradicionales, contribuyendo así, como mecanismo adaptativo, a la estabilidad social de esas comunidades, minimizando el desorden y confusión que el cambio sociocultural produce en sus individuos.

Al mismo tiempo el asociacionismo cumple también en estos ámbitos rurales y campesinos una importante función al paliar o suplir, al menos en parte, la precariedad de la atención de las instituciones del estado a los mismos, centradas fundamentalmente en los núcleos urbanos estratégicos desde el punto de vista de los sistemas económicos, sociales y políticos que constituyen la estructura básica de dicho estado, con lo que las zonas periféricas con respecto a esos núcleos y sistemas se ven desprovistas de buena parte de los servicios, prestaciones y mecanismos ofrecidos por el mismo, lo cual promueve como respuesta el desarrollo de instancias marginales o colaterales, a través de las que se intentan suplir las citadas carencias, que pueden ser desempeñadas, al menos enparte, por asociaciones y agrupaciones por medio de las cuales pueden «institucionalizarse», crearse y extenderse relaciones de patronazgo, clien- 
telismo y alianza, actuando como vehículos de intermediación con los poderes e instituciones del estado y con los centros socio-económico-políticos de los que dependen las comunidades rurales, ofreciendo canales a los individuos en sus relaciones con aquéllos.

Si esta es la visión que del asociacionismo voluntario ha predominado en la perspectiva de los antropólogos, sobre todo británicos y norteamericanos, hasta hace relativamente poco tiempo, en la Sociología, tradicionalmente mucho más interesada en el tema, sobre todo en relación al campo general del estudio de las formas de organización y de los grupos, el estudio del asociacionismo se ha limitado también principalmente al contexto de determinado tipo de sociedades urbano-industriales, en especial la norteamericana, la británica y, en cierta medida, la francesa, habiendo centrado además su interés sobre la función de las asociaciones como ámbitos de participación y ejercicio del poder en sociedades que poseen sistemas democrático-formales de organización y representación política -enmarcando su análisis, por lo tanto, en una perspectiva pluralista y funcionalista-, así como en el carácter específico de las finalidades de las formas asociativas, y en su naturaleza esencialmente «utilitaria» $^{13}$.

Ambas interpretaciones del asociacionismo se revelan como inadecuadas, limitadas e insuficientes para comprender las formas, papeles y funciones que las asociaciones presentan y desempeñan en sociedades con situaciones distintas a las que caracterizan a las tomadas como campo de estudio tanto por la Antropología, como por la Sociología «clásicas», y en concreto para abordar el estudio del asociacionismo en Andalucía. La aplicación mecánica de modelos teóricos elaborados a partir de la observación del asociacionismo en situaciones específicas pueden dar lugar a interpretaciones erróneas al ser aplicados en otras situaciones diferentes.

Con respecto a la interpretación antropológica funcionalista, y aún aceptando que el asociacionismo voluntario cumple una función de adaptación de los individuos al cambio sociocultural y a la transformación de las estructuras e instituciones tradicionales, en Andalucía, como en otras áreas y países del ámbito capitalista desarrollado, no es ésta la función más importante. El énfasis en el papel adaptativo tenía su fundamento en la situación de profundo cambio sociocultural y de desarraigo a los que se veían sometidas las sociedades en las que primeramente se estudió el asociacionismo, ligado a procesos de emigración campo (tribu)-ciudad. Formas asociativas basadas en buena medida en patrones «tradicionales» de relación y cooperación, tendentes esencialmente a reproducir los sistemas de vida tribales a través de su adaptación a las nuevas realidades urbanas, aunque también pudieran jugar un importante

13. SMITH, C. y FREEDMAN, A. Op. cit.; SILLS, D.L. Op. cit., 1974. 
papel en los procesos de aculturación y asimilación de los sistemas de vida urbanos por parte de sus integrantes.

Esta interpretación podría ser válida, con muchas matizaciones, para analizar fenómenos como el de la proliferación de asociaciones andaluzas en zonas de emigración masiva, como es el caso de Cataluña, Madrid o el País Vasco, pero en absoluto para explicar el asociacionismo interno que se da en Andalucía, sociedad de antiquísima tradición urbana en la que, a peasr del proceso de emigración «campo-ciudad» que se produce en las décadas de los años cincuenta y sesenta con especial intensidad, no ha existido una dicotomía urbanorural, industrial-agraria o campesina, radical y profunda, salvo en algunas zonas marginales; sociedad en la que las asociaciones existentes tienen un fuerte carácter local, siendo prácticamente inexistentes o de muy débil carácter, las asociaciones o federaciones supracomunales, y donde las asociaciones constituyen, sobre todo, marcos para la interrelación social, medios para la obtención de prestigio y para acceder, mantener o ampliar posiciones de liderazgo, instrumentos, en definitiva, utilizados para el ejercicio del control del poder social sobre y dentro de la comunidad local.

Con respecto a la interpretación sociológica, tenemos en primer lugar que, frente a las finalidades específicas y al carácter inmediatamente utilitario que, según ella, serían rasgos esenciales de las formas asociativas existentes en las sociedades sobre las que han realizado principalmente sus análisis los sociólogos, la mayor parte de las formas asociativas andaluzas poseen un carácter multifuncional por debajo de su finalidad concreta explicitada formalmente en su denominación y estatutos. Así mismo, la pretendida «debilidad» del asociacionismo voluntario en Andalucía se basa en la aplicación sobre el particular de modelos exóticos a la realidad sociocultural andaluza y de criterios cuantitativos que atienden casi exclusivamente al número de asociaciones por habitante - lo cuál, por otra parte, tampoco marca una diferencia tan abismal como algunos han llegado a establecer ${ }^{14}$, tal como hemos tenido ocasión de comprobar en nuestra investigación- sin tener en cuenta aspectos tan importantes o más para el establecimiento de la dimensión real del fenómeno asociativo, como son el grado de participación de los individuos en las asociaciones más allá de su adscripción formal, el nivel de concentración de la asociatividad (una sola asociación multifuncional puede incluir un número de miembros igual o superior al de tres o cuatro asociaciones de finalidades específicas juntas), la extensión de la influencia de las asociaciones y sus papeles de protagonismo en el desenvolvimiento de la vida de la comunidad en la que se hallan insertas, o la relación de las asociaciones con las estructuras, sistemas e iustituciones sociopolíticos de la citada comunidad, entre otros muchos.

14. LINZ, J. Op. cit. 


\subsection{Las cofradías y hermandades}

A ambas interpretaciones «clásicas» del asociacionismo voluntario, la antropológica y la sociológica, se les escapa la importancia fundamental que como formas asociativas tienen las cofradías y hermandades en Andalucía. A la interpretación antropológica debido principalmente a la inexistencia o características muy diferentes de este tipo de asociaciones de carácter formal «religioso-ceremonial» en las sociedades en las que tradicionalmente trabajaron los antropólogos y especialmente en las que por primera vez se abordó el estudio del asociacionismo. A la sociológica por considerarlas como formas asociativas de carácter exclusivamente religioso y prácticamente como elementos residuales de situaciones anteriores, como formas de agrupamiento características de las sociedades estamentales.

Las cofradías y hermandades andaluzas, lejos de ser instituciones residuales y más allá de sus finalidades formalmente religiosas, cumplen y desarrollan una multiplicidad de funciones sociales al menos de tanta importancia, si no más, que las estrictamente religioso-ceremoniales que son las que dan motivo a su existencia en primera instancia. Hasta tal punto es así que hermandades y cofradías han sido, y en muchos casos siguen siendo, las únicas formas asociativas existentes en muchas pequeñas poblaciones de Andalucía. Importancia además que, lejos de verse atenuada por el proceso creciente de «modernización», se encuentra en plena expansión, como lo demuestra la continua creación de nuevas entidades de este tipo, incluso de manera paralegal con respecto a la institución eclesiástica; y como lo demuestra también la participación masiva en las mismas, no sólo ni principalmente de modo formal, como socios inscritos, sino a través de la identificación con ellas de individuos y grupos de prácticamente todos los sectores de la sociedad andaluza. Significación que se pone de manifiesto en el interés que la presencia en y la dirección de las hermandades y cofradías han despertado siempre, y hoy de manera especialmente notable, entre los miembros de los grupos dominantes económica y socialmente y dirigentes políticamente de dicha sociedad.

Lo cierto es que las hermandadses y cofradías constituyen las formas asociativas de mayor implantación en Andalucía, sin paralelo con ninguna otra sociedad, hecho que explica, al menos en parte, la menor preencia de otro tipo de asociaciones, sobre todo en muchas comunidades «no urbanas», en los pueblos de menos de 10.000 habitantes de buena parte de Andalucía.

El olvido de la dimensión real del fenómeno asociativo representado por las hermandades y sus implicaciones en los sistemas de relaciones sociales y de poder locales - también en las hermandades se da un fuerte localismo, incluso cuando se constituyen en relación a fenómenos simbólico-ceremoniales de carácter supracomunal, como pueden ser El Rocío, la Virgen de la Cabeza 
de Andújar, entre otros, las hermandades siguen teniendo un ámbito local o hasta grupal (son hermandades filiales pero sin perder en ningún momento su identidad local) - es uno de los factores que explican la errónea interpretación del asociacionismo andaluz desde determinadas perspectivas.

Consideramos que los fenómenos y procesos de la vida social deben ser analizados en relación al contexto de las formaciones económico-sociales y de las etnicidades específicas, o al menos que las conclusiones de dichos anáhisis deben ser contrastados en formaciones sociales diferentes a las que sirvieron como campo de observación para su elaboración, antes de proceder a la formulación de explicaciones generales sobre los citados fenómenos y procesos. Desde nuestro punto de vista, las formas asociativas representadas por las hermandades y cofradías constituyen uno de los rasgos fundamentales que, en el aspecto de las formas de organización y agrupamiento social y de la expresión de la sociabilidad, más claramente personalizan a la formación social andaluza, lo que las convierte en uno de los marcadores más significativos y diferenciadores de su etnicidad, constituyendo además un claro ejemplo de cómo un elemento, una institución originada en el contexto de un modo de producción precapitalista, no sólo no desaparece con el proceso de transición (subsunción real) y establecimiento (subsunción formal) del modo de producción capitalista como dominante, sino que, manteniendo una forma aparente muy poco alterada, se transforma en sus funciones latentes y en sus sistemas de relaciones subyacentes, convirtiéndose, no ya en un elemento residual, sino en un componente central de la nueva formación social resultante, de importancia fundamental para el mantenimiento y reproducción de la misma y del modo de producción dominante a cuyas leyes fundamentales responde ${ }^{15}$.

\subsection{Casinos y peñas}

Con una extensión e implantación menores que las hermandades y cofradías, pero con una destacada significación en la vida y los sistemas de relaciones sociales de muchas ciudades y pueblos andaluces, encontramos la presencia de asociaciones de finalidad formal «recreativo-cultural», con una función explícita como instituciones para la «ocupación del ocio y el tiempo libre», como son los casinos, los círculos, las peñas futbolísticas, taurinas, flamencas, y las sociedades recreativas, entre otras. Se trata de asociaciones que, más allá de sus objetivos expresos, poseen un carácter multifuncional, desempeñando

15. Camilo José Cela Conde ofrece un ejemplo muy interesante de una situación de este tipo para el caso de la formación social mallorquina (CELA CONDE, C.J. Capitalismo y campesinado en la isla de Mallorca. Madrid, 1979). 
también un importante papel como instancias para la expresión de la soćiabilidad más o menos generalizada, implicando a los integrantes de un sector social determinado o a varios, raramente al conjunto de la comunidad, y como ámbitos para el establecimiento y el desarrollo de las redes de relaciones interpersonales y de poder, tanto verticales (sistemas de relaciones patrón-cliente), como horizontales (relaciones de cooperación, amistad, alianza, solidaridad, ayuda mutua), poniéndose con ello de manifiesto la fuerte tendencia a la personalización de las relaciones sociales, identificada como uno de los marcadores más profundos de la etnicidad andaluza ${ }^{16}$, que encuentra en contextos como los ofrecidos por este tipo de asociaciones - como también por las hermandades- el ambiente más propicio para la interacción social próxima y teñida, ya sea real, ya de manera ficticia o simbólica, de connotaciones afectivas.

Desde este punto de vista, hermandades y cofradías, casinos y peñas, formas de asociacionismo absolutamente mayoritarias en Andalucía, constituyen instituciones políticas de notable relevancia en la vida de las ciudades y pueblos andaluces. Instituciones políticas en el sentido amplio del término, como instancias que sirven de campo de acción para el establecimiento y desarrollo de las relaciones de poder entre los individuos y los grupos sociales, y como instrumentos para el logro del liderazgo y del control de dicho poder social. Poder entendido también en su sentido sociocultural más extenso, como capacidad de influencia y orientación de la opinión y de la actividad de la mayoría de los integrantes de un grupo, y no necesaria ni exclusivamente como autoridad político-administrativa, aunque con frecuencia lo uno conduzca a lo otro de manera más o menos directa.

Los casinos y círculos surgidos en la segunda mitad del siglo XIX y durante el primer tercio del $\mathrm{XX}$, por ejemplo, tendrán en muchos casos un carácter explícitamente político, o lo adquirirán en determinados momentos, siendo en la mayoría de las ocasiones entidades asociativas, más o menos abiertas, pero casi siempre controladas y al servicio de las élites locales, que las utilizarán como medios para el establecimiento, mantenimiento y reproducción de los sistemas de relaciones de naturaleza patrón-clientelista y de los sistemas caciquiles de dominación sociopolítica.

Las transformaciones socioeconómicas que han tenido lugar a lo largo de los últimos 25 ó 30 años en Andalucía, han determinado el debilitamiento de los sistemas de relaciones sociales anteriormente vigentes, el desarrollo de sectores sociales intermedios, cuyas ocupaciones y formas de vida les permitirán cierta autonomía con respecto a los elementos de los grupos dominantes locales (pequeños industriales y comerciantes, profesionales, empleados, trabaja-

16. MORENO NAVARRO, I. «Hacia la generalización de la conciencia de identidad (19361983)». Historia de Andalucia. Vol. VII, Barcelona, 1984, pp. 355-378. 
dores especializados autónomos o por cuenta ajena), se debilitarán notablemente las situaciones de fuerte dependencia personal sobre las que se montaba el sistema de patronazgo. Ello ha determinado, así mismo, la transformación de los casinos y círculos tradicionales, que han experimentado una apertura a nuevos sectores sociales y han visto el acceso a sus puestos directivos de los miembros de los sectores sociales en ascenso; o que, por el contrario, han sufrido una decadencia y degradación notables, que les ha llevado en muchos casos a su desaparición. Y al mismo tiempo, se produce la extensión de otras formas asociativas, también de carácter formal «recreativo-cultural», representativas de un modelo de sociabilidad más abierto y «popular», distinto del que caracterizaba a los casinos tradicionales, siendo entidades que responden específicamente a las nuevas condiciones sociales, proporcionando a los miembros más activos de los sectores con mayor dinamismo en la sociedad local vías para el acceso al prestigio y al liderazgo social a través del desarrollo de sus redes de relaciones, ya no basadas en posiciones de patronazgo fundamentalmente, sino en estrategias de alianzas e intereses mutuos, de carácter mucho más flexibles e inestable que las tradicionales de tipo clientelista, para lo cuál estas formas asociativas se muestran particularmente propicias.

En el aspecto político, la escasísima afiliación que caracteriza a los partidos y el carácter fuertemente personalista que tiene la política local en Andalucía $\rightarrow$ sobre todo en las comunidades no urbanas-, estas asociaciones han desempeñado y desempeñan en muchos casos los canales a través de los cuáles (y en bastantes ocasiones a partir de los cuáles) se han seleccionado y potenciado a los líderes de diferentes opciones políticas partidistas, y se ha movilizado a los sectores de la población que constituyen el campo potencial de votantes de cada una de ellas.

\section{EL ASOCIACIONISMO ALJARAFEÑO}

Nuestra investigación ha pretendido verificar las hipótesis interpretativas expuestas con anterioridad a través del análisis de las formas asociativas existentes en un ámbito sociocultural específico, como el que constituye la comarca del Aljarafe sevillano, cuyos rasgos socioeconómicos peculiares y la influencia notable ejercida sobre ella por la ciudad de Sevilla, con la que mantiene desde antiguo estrechas relaciones de todo tipo, la dotan de un gran interés para el análisis del fenómeno que nos interesaba: las características de las citadas formas asociativas, sus formas de estructuración, de expresión y funcionamiento, sus substratos económicos y sociales, sus bases institucionales tradicionales, sus funciones con respecto a los procesos de identidad y a los sistemas sociopolíticos locales, etc. 
Según conocemos en base a los estudios antropológicos de carácter monográfico realizados en distintas comunidades de la comarca (I. Moreno Navarro, 1972; E. Aguilar Criado, 1983, entre otros), la estructura socioeconómica que la caracteriza de manera general está configurada esencialmente por un sistema de distribución de la propiedad de la tierra relativamente poco concentrada, con la existencia de un sector importante, cuantitativa y, sobre todo, cualitativamente, de pequeños y medianos propietarios agrícolas y, consiguientemente, con una relativa menor importancia y significación del proletariado agrario, así como también con el escaso peso, comparativamente con otras comarcas (fundamentalmente dentro del Valle del Guadalquivir), de la clase de los terratenientes. Estructura socioeconómica que parece ser la base explicativa de la existencia en la comarca, al menos en un porcentaje de comunidades de la misma lo suficientemente elevado como para ser casual, de un sistema dual de organización socio-ceremonial, observable de forma explícita a nivel simbólico-ritual, que da lugar a una división, más o menos vertical, simétrica y equilibrada de la comunidad; división que se superpone a la determinada por la fundamental, definida por la existencia de las clases sociales, las cuáles son negadas u ocultadas simbólicamente a través de aquélla.

A este respecto, uno de los objetivos de nuestra investigación sobre el asociacionismo aljarafeño fue comprobar si la referida estructura dicotómica de carácter simbólico-ceremonial era reproducida, en qué medida y con qué peculiaridades por otras formas asociativas y en otros contextos de expresión de la sociabilidad que no fueran los representados por las hermandades y los contextos festivos; o si, por el contrario, la configuración de las formas asociativas que nos interesaban respondían a principios diferentes e incluso opuestos a los determinados por la división dual del grupo, como pudiesen ser la sociabilidad generalizada, los intereses de clase, los socio-profesionales, los económi$\cos$, los políticos, etc. de modo más explícito y directo. Por otra parte, nos interesaba también comprobar la veracidad de la hipótesis según la cuál la existencia en una determinada comunidad de un sistema dual de hermandades-mitades, produciría un vaciamiento y desfuncionalización, cuando no un bloqueo, de las formas asociativas no religioso-ceremoniales que pudiesen darse en dicha comunidad, en comparación con otras en las que no se diese dicho sistema, debido a la acción de absorción y monopolización de la expresión de la sociabilidad operada por el mismo, el cuál acaparararía las necesidades asociativas de sus miembros, ofreciendo un campo muy atractivo para el desarrollo de las relaciones interpersonales a través de un esquema de identidad-afinidad y enfrentamiento-rivalidad. En resumen, nos proponíamos comprobar la presunción de que, en principio y en comparación con el conjunto de la comarca, era en las comunidadses en que la citada división en mitades socio-ceremoniales opera con mayor fuerza (como son los casos de Huévar, Benaca- 
zón, Mairena, Carrión, Albaida, Aznalcázar), donde se observa el fenómeno de que, sin llegar a impedir de modo absoluto la existencia de otras formas asociativas, son ellas las que realmente absorven la mayor parte de la atención y la sociabilidad de sus miembros, vaciando de contenido y significación como instancias para la expresión de la sociabilidad a las que han podido subsistir frente a la «competencia» de las hermandades-mitades, estando aquéllas limitadas además a fines muy concretos - asociaciones de padres de familia, de padres de alumnos, de deficientes mentales, etc. - fuera de los cuáles no ejercen influencia real importante sobre la vida social y las relaciones interpersonales dentro de la comunidad.

Otro de los aspectos abordados en nuestra investigación fue el análisis de las relaciones entre las formas y estructuras de la sociabilidad asociativa, en especial las representadas por los casinos, peñas y sociedades recreativas, y las redes y campos sociales establecidos a nivel local y supralocal, así como con las estructuras de poder y los sistemas de patronazgo-clientelismo operantes a los mismos niveles y en sus conexiones con el exterior de la comarca, con los centros económicos, sociales y político-administrativos de los que depende. A través de todo este entramado de instituciones, asociaciones y principios formales, es decir concretados en grupos o estructuras particualres, o informales, como formas de comportamiento, redes sociales, círculos de amistad, etc., hemos intentado dibujar las líneas básicas de la malla social de relaciones interpersonales e intergrupales, la cuál constituye el núcleo fundamental que determina la identidad sociocultural de un grupo o comunidad como tal y su integración en un ámbito comarcal más amplio con el que comparte dichas líneas generales y las mismas fórmulas y sistemas de expresión de la sociabilidad de sus miembros.

\section{EL PROCESO ASOCIATIVO EN EL ALJARAFE}

Nos detendremos a continuación en la descripción de algunos de los rasgos fundamentales que encontramos en el asociacionismo aljarafeño, en el planteamiento de las condiciones socioeconómicas que subyacen bajo las formas del mismo, y en el establecimiento de las líneas básicas del proceso experimentado por dicho asociacionismo, con la intención de que ello pueda servir como ilustración de los postulados anteriormente planteados.

En primer lugar una constatación: la práctica total ausencia del fenómeno asociativo voluntario en el Aljarafe en época anterior a 1850 , como mínimo, a excepción naturalmente de las cofradías y hermandades, las cuáles acumulaban tanto las funciones religioso-ceremoniales explícitas características de este tipo de entidades, como otras de carácter social, asistencial, profesional, ac- 
tuando también como asociaciones de interses comunes y como ámbitos para la expresión y el desarrollo de la sociabilidad, entre otras. Su número era mayor que en la actualidad, especialmente en términos relativos, y su actividad estaba igualmente bastante más integrada en la vida cotidiana de las distintas comunidades, y no casi exclusivamente en los contextos festivo-ceremoniales como en el presente. Muchas de ellas tenían importantes funciones como asociaciones de socorros mutuos, funerarias, asistenciales. Estas asociaciones socio-religiosas respondían a esquemas y estructuras propios de una sociedad precapitalista, estamental en cuanto a su forma de organización social. No es extraño, por lo tanto, que el desmoronamiento de dicho tipo de sociedad, operado no obstante con retraso considerable respecto a los centros urbanos en los medios rurales y campesinos, tuviese como una de sus consecuencias la decadencia, desaparición o, en algunos casos, transformación de las funciones implícitas de las hermandades y cofradías. Será en aquellos lugares en que pasaron a encarnar a las mitades socio-ceremoniales (no tenemos datos que nos indiquen su existencia durante el Antiguo Régimen, al menos con las características y funciones que las definen actualmente) donde se producirá un mantenimiento o incluso incremento notable de su importancia como instituciones fundamentales en la vida social de dichas comunidades.

La ausencia de un asociacionismo «civil» de importancia fuera del marco religioso-ceremonial, con los matices que se han señalado, se explica, como ya hemos apuntado, por el mantenimiento de estructuras socioeconómicas características de la sociedad estamental, sin que el ocaso del Antiguo Régimen, operado a nivel estatal desde principios del siglo XIX, hubiese dado lugar a transformaciones realmente profundas en las zonas rurales y campesinas en general, andaluzas en particular y en la comarca del Aljarafe en concreto, a pesar de su proximidad y estrecha relación con un centro socioeconómico y de poder de importancia como la ciudad de Sevilla.

Unicamente a partir de mediados del siglo XIX se inicia un lento proceso de cambio económico y sociocultural en la comarca, más debido a las transformaciones que se iban operando en el núcleo sevillano - por otra parte también bastante tímidas - que por las experimentadas internamente por las estructuras socioeconómicas aljarafeñas. Resultado de este proceso será, sobre todo, la extensión y acentuación de la influencia y dependencia de y con respecto a la capital en todos los aspectos: poblacionales y demográficos (emigración a la ciudad, concentración de la población de la comarca en los pueblos más próximos a Sevilla), económicos, comerciales (integración de manera más estrecha de lo que ya lo era en las redes y sistemas del mercado), sociales, culturales, simbólicos (de este momento arranca la relación de Sevilla con El Rocío, por ejemplo), etc. 
Proceso de cambio y transformación sociocultural que se refleja, en el tema que nos ocupa, en el inicio de un tímido proceso asociativo también en estos momentos. En un principio estará limitado casi exclusivamente a las élites locales, las cuáles empezarán a constituir algunas agrupaciones a imitación de las entidades que poco tiempo antes habían surgido en la capital: casinos y círculos de carácter político-cultural que congregaban a determinados representantes de la aristocracia y la alta burguesía sevillanas, los cuáles funcionaban más como centros de relación formal que como verdaderos ámbitos de expresión de la sociabilidad generalizada.

No será hasta prácticamente la década de los años setenta del pasado siglo cuando se asista en la comarca al inicio de un movimiento asociativo de magnitud relativamente más amplia y de extracción social menos restringida a las élites socioeconómicas, surgiendo en algunos lugares (normalmente los núcleos más significativos desde el punto de vista económico, social y político-administrativo) casinos, círculos y centros con fines formales diversos: culturales, recreativos, políticos, cooperativo-asistenciales, etc. Movimiento que será paralelo a la formación de las primeras organizaciones políticas y sindicales, igualmente de muy escasa presencia en la comarca. No es extraño que sea precisamente en este período de inestabilidad socio-política de finales de la década de los sesenta y principios de los setenta cuando se produce este primer desarrollo asociativo, lo cual volverá a repetirse en los momentos sucesivos de crisis e inestabilidad que tendrán lugar a lo largo del último cuarto del siglo XIX y el primer tercio del XX. Ello nos confirma el papel señalado al asociacionismo no sólo como instancia de control, orientación, apoyo y canalización de los individuos y sus inquietudes ante situaciones de cambio sociocultural disgregadoras de las estructuras tradicionales, sino también como elemento potenciador y/o acelerador del cambio socio-político, favoreciendo el surgimiento de nuevos liderazgos y redes de relaciones de poder.

No obstante los rasgos característicos del asociacionismo de los círculos y casinos del Aljarafe contrastará con el sevillano, del que generalmente es réplica formal, en que en su mayor parte responderá a una base sociológica y de relaciones interpersonales de carácter más persistente que las que sustentaban, y en parte al menos siguen sustentando, al asociacionismo capitalino, observándose incluso en algunos casos el mantenimiento de unos mismos grupos aunque bajo denominaciones formalmente diferentes, respondiendo en su esencia a las mismas estructuras y sistemas de relaciones interpersonales.

En relación con lo anterior se observa también que, por regla general, la duración de la vida media de las asociaciones es mayor en la comarca que en la ciudad, presentando además una estabilidad relativamente mayor que las de Sevilla. 
También es observable, aunque ello sea en realidad un rasgo bastante general en todo el asociacionismo andaluz de épocas anteriores y aún de la actual, el carácter casi exclusivamente masculino de la inmensa mayor parte de las asociaciones, así como su finalidad formal como «agrupaciones para la ocupación del ocio y el tiempo libre» principalmente. Lo que contrasta, sobre todo encuanto al primer aspecto con el carácter mixto de las hermandades, en las que incluso en muchas ocasiones las mujeres desempeñan un papel muy relevante, si no a nivel formal, oficial o institucional, si en el real y de actuación, lo que se hace especialmente evidente en el caso de las hermandadses-mitades. El segundo aspecto puede ser considerado como un hecho eminentemente formal, ya que la práctica totalidad de estas asociaciones actúan en realidad como organizaciones multifuncionales, como marcos para el desarrollo de la sociabilidad generalizada y las relaciones interpersonales en sentido amplio.

La guerra civil y sus consecuencias tendrán una repercusión muy notable sobre el asociacionismo a nivel general de todo el estado, y en particular en la comarca. Se produce la desaparición y el desmantelamiento de numerosas asociaciones, viéndose limitadas las que lograron sobrevivir o las muy pocas que se crearon en la posguerra por fortísimos condicionamientos políticos, sociales e ideológicos que, en la práctica, darán lugar a la perdida casi total de su funcionalidad como marcos para la expresión y el desarrollo de la sociabilidcad de la población. Curiosamente este período coincidirá con un momento de revitalización de las hermandades y cofradías, así como el de otras asociaciones religiosas de menor importancia, como las Hijas de María, Acción Católica, etc., bajo cuyos fines expresamente religioso-ceremoniales se pudo albergar, al menos en parte, la expresión y el desenvolvimiento de la sociabilidad, muy limitada en sus formas y ámbitos de desarrollo por la dictadura, aparte de otras causas de índole política e ideológica.

El desarrollo económico general y la aceleración del proceso de urbanización experimentados, sobre todo en la década de los años sesenta, y la influencia de dichos procesos sobre las zonas rurales y campesinas y en la comarca del Aljarafe de modo particular, con los consiguientes cambios y transformaciones socioeconómicas que ello originará - cuya profundidad e intensidad serán especialmente notables en esta comarca, dada su proximidad y estrecha relación con Sevilla - dará lugar a un nuevo impulso del asociacionismo, poniéndose nuevamente de manifiesto la fuerte conexión entre cambio y crisis sociocultural y económica y fenómeno asociativo, así como su relación y conexión con el exterior de la comarca, representado por las estructuras, sistemas, instituciones y mecanismos del estado y de los poderes socioeconómicos, políticos y administrativos que influyen, inciden y determinan sobre la vida de las comunidades aljarafeñas. 
En la actualidad, desde principios de los años setenta, y como fruto de la transformación de la naturaleza del estado, el fenómeno asociativo cobrará un nuevo auge, aunque los profundos cambios socioeconómicos operados en la comarca y en sus formas de vida tradicionales, han dado lugar paralelamente al cambio y transformación de muchos de los aspectos que caracterizaban al asociacionismo hasta épocas recientes, en concreto la decadencia bastante generalizada, aún con excepciones, de los casinos, y el desarrollo y multiplicación de otro tipo de asociaciones, como las peñas deportivas y futbolísticas, o las peñas flamencas. En esto ha tenido una decisiva influencia el desarrollo de los medios de comunicación y la involucración de las comunidades de la comarca en los fenómenos característicos que distinguen las formas asociativas aljarafeñas de las existentes en los centros socio-políticos y económicos, fundamentalmente debido al reducido tamaño de las comunidades de la comarca, así como a la fuerza que, por ello principalmente, continuan teniendo las relaciones interpersonales directas, cara a cara y continuadas.

\section{JUSTIFICACION FINAL}

En las páginas que anteceden sólo hemos pretendido apuntar algunas cuestiones relativas a la significación y las funciones socio-políticas de determinadas formas asociativas, cuya relevancia, más allá de sus funciones, hemos constatado en nuestra investigación sobre el asociacionismo aljarafeño. Lo cuál es un rasgo extensible al conjunto de la sociedad sevillana y andaluza, lo que convierte a estas formas asociativas en marcadores muy importantes de la identidad cultural de las mismas en el terreno de la sociabilidad. Formas asociativas cuyas implicaciones sociopolíticas no han sido suficientemente valoradas debido al prejuicio de su carácter formal religioso-ceremonial, o recreativo-cultural, finalidades aparentemente alejadas de los contextos en los que se desenvuleven las relaciones sociales y de poder en el seno de las comunidades locales. Por el contrario, nuestro trabajo pone de relieve, creemos, la importancia de las funciones implícitas de dichas asociaciones, que las convierte en potenciales instrumentos para el desarrollo y la vertebración de la sociedad civil andaluza, de los que adolece y que parece imprescindible conseguir para lograr una sociedad más avanzada y participativa.

Aparte del interés por el conocimiento de una parcela de la realidad sociocultural andaluza prácticamente virgen para las ciencias sociales, uno de los motivos principales que nos han movido a estudiar el asociacionismo ha sido el de aportar nuestra modesta contribución a la profundización del conocimiento de los marcadores fundamentales que definen la identidad de Andalucía como pueblo con una cultura específica diferenciada, así como el de inten- 
tar proporcionar a los agentes sociales elementos de juicio y de análisis para el desvelamiento de uno de los mecanismos a través del que se sustenta y reproduce el control social y se desarrolla la acción política en el seno de la sociedad local, y para, en su caso, proveer de instrumentos a dichos agentes sociales para la práctica y la intervención orientadas al desarrollo de nuestra sociedad civil, entendida en el sentido gramsciano, para el fomento de la participación ciudadana y para la extensión de la conciencia de etnicidad en nuestro pueblo. 